\title{
Effect of Reverse Bias Voltages on small scale gridded CIGS Solar Cells
}

\author{
Soheyl Mortazavi ${ }^{1}$, Klaas Bakker ${ }^{2,3}$, Jorne Carolus ${ }^{4}$, Michael Daenen ${ }^{4}$, Gabriela de Amorim Soares ${ }^{1}$, Henk \\ Steijvers ${ }^{1}$, Arthur Weeber ${ }^{2,3}$, Mirjam Theelen ${ }^{1}$ \\ ${ }^{1}$ TNO Solliance, Thin Film Technology, High Tech Campus 21, 5656 AE Eindhoven, The Netherlands \\ ${ }^{2}$ ECN Solliance, High Tech Campus 21, 5656 AE Eindhoven, The Netherlands \\ ${ }^{3}$ Delft University of Technology, Photovoltaic Materials and Devices, 2628 CD Delft. The Netherlands \\ ${ }^{4}$ Hasselt University, Instituut voor Materiaalonderzoek, Wetenschapspark 1, 3590, Diepenbeek, Belgium
}

\begin{abstract}
Partial shading on photovoltaic modules can cause cells to operate at reverse bias conditions. An innovative experimental setup with two test protocols has been designed to simulate the effect of reverse bias voltage on small scale CIGS solar cells with a metal grid. In these tests, the effect on the performance of the solar cells exposed to either a stepwise increasing or prolonged constant negative bias voltage was studied. It was demonstrated that performance loss occurred mainly due to a reduction in shunt resistance. Moreover, some solar cells have partially recuperated after light soaking.
\end{abstract}

Index Terms - CIGS, reliability, partial shading, reverse bias voltage,

\section{INTRODUCTION}

Partial shading on photovoltaic (PV) modules causes reverse bias on cells which can result in temporary and permanent damages. Thin Film (TFPV) technologies often differ from conventional wafer based crystalline Silicon (c-Si) PV due to the use of monolithically interconnections resulting in long narrow cells. Especially for these modules the magnitude of reverse bias is dependent on the shape of the shade, geometrical structure of the TFPV module and the intensity of the shadow [1]. For wafer based modules bypass diodes can be tailored for optimal protection against reverse bias damage [2]. Major disadvantage for the use of bypass diodes in a string of cells is that the bypass diode bypasses the contribution of the whole string to the power output of the module. This results in loss of output power of the whole string when only a fraction of the string is shaded. The monolithically interconnection scheme used in most thin film modules does not easily allow the integration of bypass diodes therefore other means to protect from or mitigate against reverse bias damage are needed.

Off all the thin film technologies $\mathrm{Cu}$ (In, Ga)Se $\mathrm{Se}_{2}$ (CIGS) is one of the most promising with high efficiencies and growing sales volumes. However worst case scenario shading tests like in the IEC 61215 standard on CIGS modules showed that even very short events $(\sim 20 \mathrm{~s})$ can result in a permanent relative decrease in efficiency from $4 \%$ to $14 \%$ [3].

In literature, most partial shading tests have been performed on CIGS modules rather than individual cells, which resulted in the formation of hot spots and wormlike defects [3]-[7] Additionally, from literature it has been observed that light soaking can have a recuperating effect on CIGS modules after reduction in performance due to partial shading [4],[8].

Several different studies of the reverse current voltage (IV) characteristics of CIGS solar cells and modules have been executed, experimental conditions that have been varied include the influence of light intensity [8], light spectrum [8],[9], temperature [9], sample design and composition (i.e. absorber and buffer layers thicknesses) [9] on reverse bias characteristics of solar cells. As stated by Silverman et al. [3], more information is required to be able to predict and prevent degradation due to reverse bias and to optimally profit from the light soaking effect. Currently, it is expected that the geometrical structure of solar cells, magnitude of the reverse bias, the exposure time, the relaxation time, as well as the sequence of exposures all also influence the effect of reverse bias on CIGS solar cells. However, information about the exact influence of these parameters is not yet sufficiently available. It is important to understand the effects of all these parameters on individual cells in order to effectively mitigate the effects of reverse bias in modules.

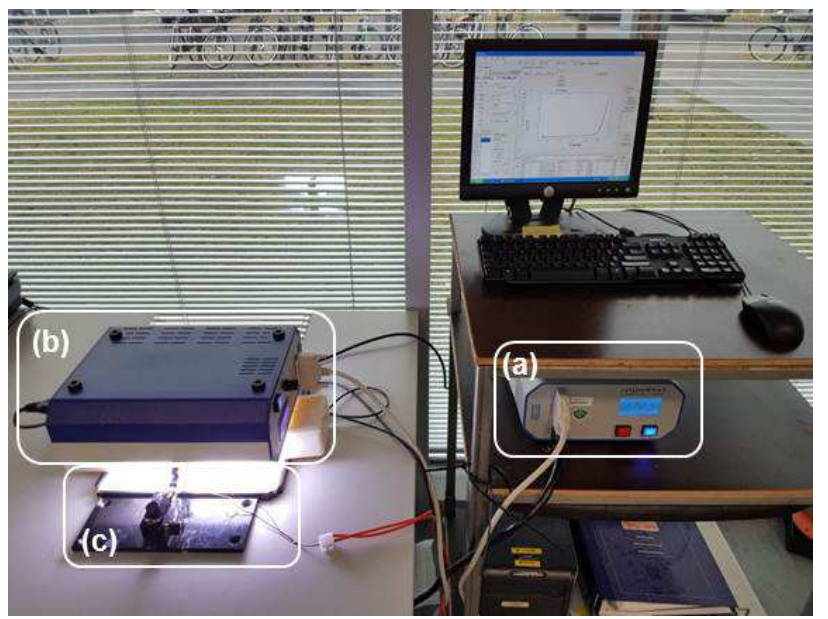

Fig. 1. (a) Iviumstat programmable potentiostat/galvanostat as central control device (b) IviSUN LED light source (c) Sample and Kelvin contact probes 
The aim of this study was to define the effect of reverse bias voltages and subsequent light soaking on gridded CIGS solar cells. A setup was designed and built to investigate the parameters that influence the reverse bias behaviour, and later to define the occurring degradation mechanisms. Initial results including the influence of magnitude and exposure time of reverse bias voltage as well as the effect of light soaking obtained with this setup are presented here.

\section{MATERIALS AND MeTHODS}

\section{A. Experimental units}

In order to execute the experiments, a commercial programmable potentiostat/galvanostat Ivium - Iviumstat has been used as the central control device of the setup. The Iviumstat is a 4-quadrant voltage/current controlled power supply that enables both forward and reverse biasing and precise four wire measurements up to $\pm 10 \mathrm{~A}$ and $\pm 10 \mathrm{~V}$ with $0.2 \%$ accuracy. (fig. 1a)

To perform measurements under illumination, a light source Ivium - IviSUN controlled by the Iviumstat has been added to the setup. It features, white LEDs $(5000 \mathrm{~K})$ with an illuminated area of $150 \times 150 \mathrm{~mm}^{2}$ (fig. $1 \mathrm{~b}$ ). Due to the precise control of voltage, current, and light intensity during defined time intervals, the Iviumstat combined with the IviSUN gives the freedom to design different test protocols for different experiments.

A cooling fan has been used to continuously blow air on the surface of the cells under test to keep them at room temperature. Kelvin probes have been used to connect the cells to the Iviumstat. (fig. 1c)

For better readability all references with respect to reverse bias voltages are based on the absolute value e.g. $-2 \mathrm{~V}$ is a lower reversed bias voltage than $-3 \mathrm{~V}$.

Due to the low intensity and non-ideal spectrum for CIGS of the LEDs utilised by the IviSUN, the intensity was estimated by comparing current densities obtained from IV and EQE measurements at standard test conditions of cells on the same substrate. This intensity of the IviSUN was roughly estimated to be 0.3 sun equivalent for the cells used and is assumed to be constant. All reported currents are calculated to the 1 sun equivalent. Series and shunt resistances are estimated from the slope at respectively the highest and lowest voltages of the IV scan that ranged from -0.3 till +0.7 Volts.

Illuminated Lock In Thermography (ILIT) pictures were made with a system from Infratec using IR LEDs for excitation. Real time infrared movies have been taken with a high speed IR camera (FLIR X6580sc) combined with a Keithley 2400 source measure unit.

\section{B. CIGS solar cells}

Non-encapsulated CIGS solar cells were deposited on a 1 mm thick $100 \mathrm{~mm}$ x $100 \mathrm{~mm}$ soda lime glass substrate (SLG). The stack contained $500 \mathrm{~nm}$ DC-sputtered molybdenum (Mo)
/ $2 \mu \mathrm{m}$ coevaporated CIGS / $50 \mathrm{~nm}$ chemical bath deposited cadmium sulfide (CdS) / $65 \mathrm{~nm}$ DC sputtered intrinsic zinc oxide (i-ZnO) / $260 \mathrm{~nm}$ DC sputtered aluminium doped zinc oxide ( $\mathrm{ZnO}: \mathrm{Al})$ with an ebeam evaporated metal $(20 \mathrm{~nm}$ $\mathrm{Ni}+600 \mathrm{~nm} \mathrm{Ag}+20 \mathrm{~nm} \mathrm{Ni}$ ) grid on top. The metal grid was made of two tapered fingers connected to a contact island which in total covered $2 \%$ of the active area of a cell. 162 individual solar cells of $10 \mathrm{~mm}$ x $5 \mathrm{~mm}$ were obtained by mechanical division via an automated scriber utilising a diamond tipped needle to cut through the CIGS and AZO. Formation of the back contact was done by scratching away the CIGS with a scalpel and application of $\mathrm{Ag}$ ink as contact material on the exposed molybdenum.

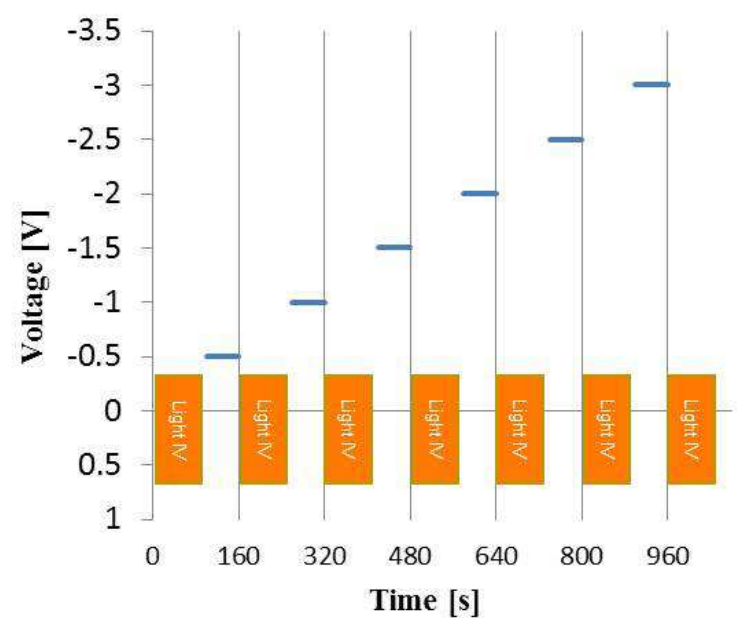

Fig. 2. Applied voltages on solar cells for the elevating reverse bias test protocol. Orange blocks indicate IV measurements under illumination $(100 \mathrm{~s})$, where the width of the block represents the duration of the measurement and the height of the block is the voltage range the IV measurements were performed $(-0.3 \mathrm{~V}$ until $0.7 \mathrm{~V})$. Blue lines are exposures to negative voltage levels $(60 \mathrm{~s})$.

\section{Treatments and methods}

To measure the susceptibility of CIGS solar cells to negative voltages, two test protocols have been defined. The first protocol was intended to evaluate the evolution of solar cell performance as a function of the magnitude of the applied reverse bias voltages and also to find if there is a fatal breakdown voltage. For this elevating reverse bias protocol, the reverse bias voltage was increased in linear steps in which the upper limit was based on preliminary dark IV measurements that showed that there is a voltage at which the cell suddenly became fully shunted. This fatal breakdown voltage occurred between -3 and -4 Volt. During the elevating reverse bias protocol, for each step the cells were exposed to a predetermined voltage level for 60 seconds in darkness. The voltage levels varied from -0.5 to $-3 \mathrm{~V}$ with $0.5 \mathrm{~V}$ increments. 

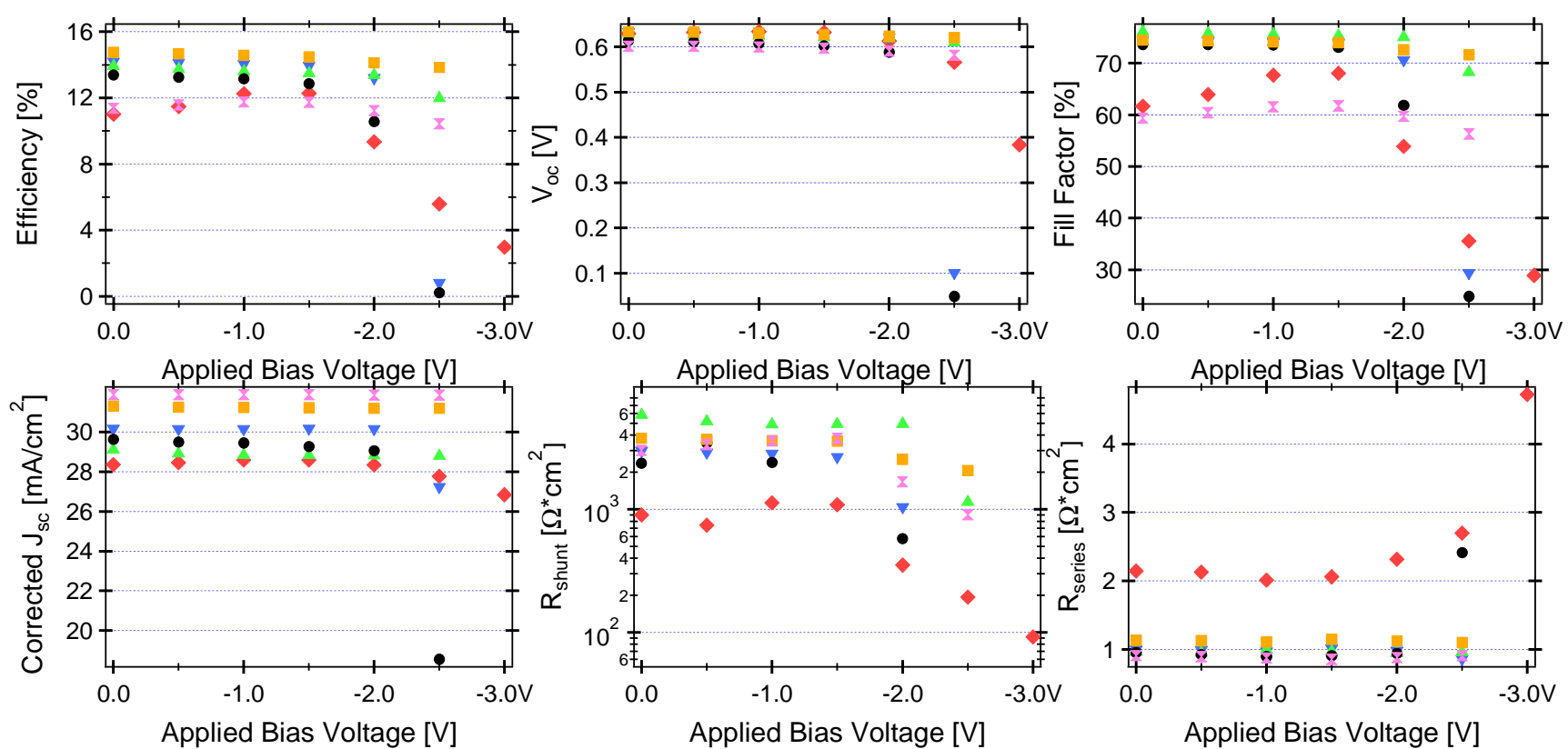

Fig. 3. IV parameters as function of applied bias voltage for 6 similar CIGS solar cells under the elevating reverse bias test protocol. Measurements were performed after cells have been exposed to the defined negative voltages for $60 \mathrm{~s}$. The $\mathrm{J}_{\mathrm{sc}}$ and the efficiency were normalized to 1 sun.

Before each step, an IV measurement under illumination was performed to quantify the evolution in electrical performance. The IV measurement was executed in both forward and reverse scan direction ranging from $-0.3 \mathrm{~V}$ to $0.7 \mathrm{~V}$ and back. The duration of the scan was 100 seconds. An illustration of the conditions applied during this test protocol is presented in fig. 2.

A second protocol has been defined to observe solar cell behaviour under elongated exposure to "mild" negative bias voltages. Two voltages were selected for this constant negative voltage protocol, the selection was made based on the results obtained from the elevating reverse bias experiment that will be described in the Results and Discussion section. For each of the 30 cycles, first an illuminated IV measurement was executed, followed by the exposure to a constant negative voltage for 120 s in darkness.

To study the effect of light soaking after the application of reverse bias voltage, the samples have been exposed to the light for 6 hours at $\mathrm{V}_{\mathrm{OC}}$ conditions. During this illumination IV measurements have been performed every 10 minutes to monitor the evolution of electrical parameters.

\section{RESULTS AND DISCUSSION}

\section{Elevating reverse bias tests}

The elevating reverse bias protocol was executed on 6 CIGS solar cells. The evolution of electrical parameters of these 6 solar cells as a function of applied voltage is shown in fig. 3 . All cells were stable to exposures to voltages smaller than $-1.5 \mathrm{~V}$ and started to display a small decrease in efficiency when exposed to $-2 \mathrm{~V}$ for 60 seconds, while larger decreases in electrical parameters became visible after exposure to $-2.5 \mathrm{~V}$. Further increasing the voltage to $-3 \mathrm{~V}$ was fatal for five out of six cells. The main reason for the observed reduction in efficiency was the decrease of shunt resistance. The dramatic reduction in shunt resistance was likely also influencing other electrical parameters especially $\mathrm{V}_{\mathrm{oc}}$. The cell that survived the exposure to $-3 \mathrm{~V}$ was later exposed to light soaking (not depicted). This cell displayed an increase of efficiency in absolute value from $3.0 \%$ to $7.2 \%$. This recuperation was mainly the result of increase in shunt resistance $\left(91 \Omega . \mathrm{cm}^{2}\right.$ to $\left.264 \Omega . \mathrm{cm}^{2}\right)$ as well as in $\mathrm{V}_{\text {oc }}(0.38 \mathrm{~V}$ to $0.6 \mathrm{~V})$. None of the
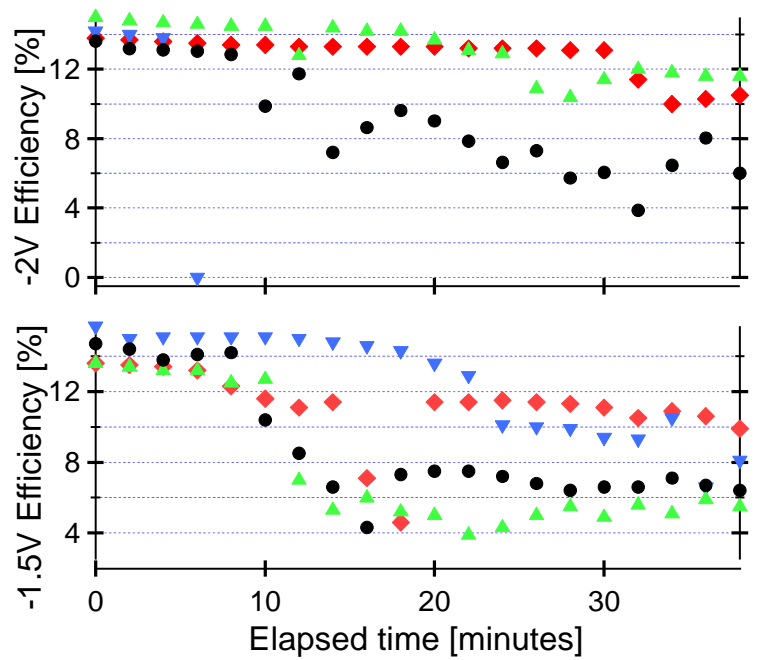

Fig. 4. Efficiency evolution for first 20 cycles for both constant negative voltage exposures to $-2 \mathrm{~V}$ (top graph) and $-1.5 \mathrm{~V}$ (bottom graph). 

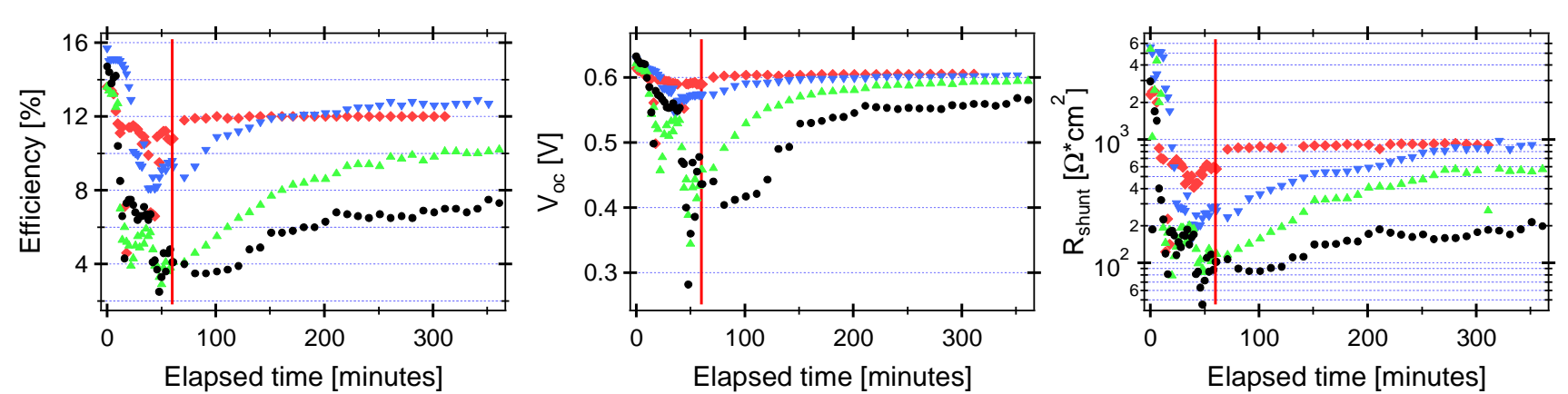

Fig. 5. IV parameters evolution of the 4 CIGS solar cells under constant negative voltage test protocol, exposed to $-1.5 \mathrm{~V}$ for 120 seconds after each IV measurement. The red line indicates when light soaking started.

other cells showed any improvement after light soaking.

\section{Constant reverse bias tests}

From the results of the previous experiment two voltages were selected:

- $-1.5 \mathrm{~V}$ which was the highest voltage that cells were stable for $60 \mathrm{~s}$ and

- $-2 \mathrm{~V}$ this was the first voltage to exhibit major changes in IV parameters.

For both voltages 4 similar samples were tested. The evolution of efficiency during the first 20 cycles for exposure to both $-1.5 \mathrm{~V}$ and $-2 \mathrm{~V}$ are graphed in fig. 4 . In general a strong decrease in efficiency over time was observed for all cells exposed to constant negative bias voltages. However, every cell seems to have a point of abrupt change that occurs at different elapsed times. As with the elevating reverse bias test the changes were mostly due to a decrease in shunt resistance. Most cells exposed to constant negative voltage showed a decrease in shunt resistance already after the first one to five exposures. However, for two cells exposed to $-2 \mathrm{~V}$ it took between 10 and 18 cycles to exhibit changes in FF and shunt resistance (not depicted). Interestingly, for these cells the efficiency dropped over time due to a decrease in $\mathrm{V}_{\mathrm{oc}}$. The fact that no change in shunt resistance and a change in $\mathrm{V}_{\mathrm{oc}}$ was observed could be an indication that something is happening in the diode parameters of the solar cell. Similar observations of a separate influence of shunt resistance and diode parameters on amorphous-Silicon cells have been made by Donaonkar et al. [10]. After the first changes the behaviour of electrical parameters is not fluent and displays hills and valleys. More information is displayed in fig. 5 that shows the evolution of efficiency, $\mathrm{V}_{\mathrm{oc}}$ and shunt resistance for the cells exposed to $-1.5 \mathrm{~V}$, followed by light soaking.

\section{Influence of light soaking}

The results for all IV parameters of the cells exposed to $-1.5 \mathrm{~V}$ and consecutive light soaking over time are shown in fig. 5 . The red line in fig. 5 indicates the transition from the exposure to negative bias of $-1.5 \mathrm{~V}$ to light soaking at $\mathrm{V}_{\mathrm{oc}}$. Not shown is that the trend for both -1.5 and $-2 \mathrm{~V}$ are roughly the same.
The reduction in efficiency due to the exposure to negative bias is partly restored by the light soaking. Cells that have been severely damaged did not recuperate or recuperated slowly. Recuperation is visible in all electrical parameters however none of the parameters came back to the initial value.

It should be mentioned that the $\mathrm{I}_{\mathrm{sc}}$ drops relatively about $3 \%$ after the first ten minutes and remains stable. This initial decrease is most likely due to heating of the LED light source, this drop in $I_{s c}$ however does not explain why the efficiency remains much lower than initial.

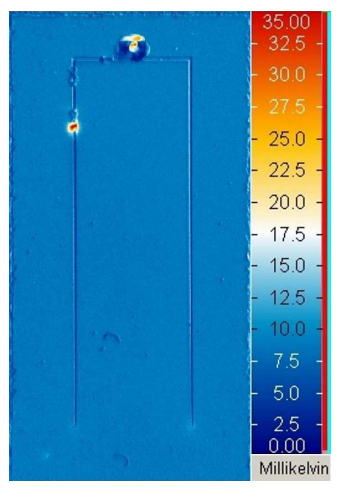

Fig. 6- Lock in Thermograph of a solar cell from the elevating reverse bias test protocol after final exposure to negative bias. Shunts are visible as red spots of increased temperature on the metal grid and contact island

\section{Identification of hotspots}

Lock-in thermography has been performed on a number of cells after exposure to both measurement protocols. It was noted that especially for the cells that stopped working during the elevating reverse bias test hotspots were formed underneath the metal grid as well as on top and surrounding the contact islands. Fig. 6 shows a typical LIT image of a cell after completion of the elevating reverse bias protocol, revealing hotspots underneath the metal grid and at the point of electrical contact. An explanation of the shunts at the contact islands was revealed when recording a live thermal 


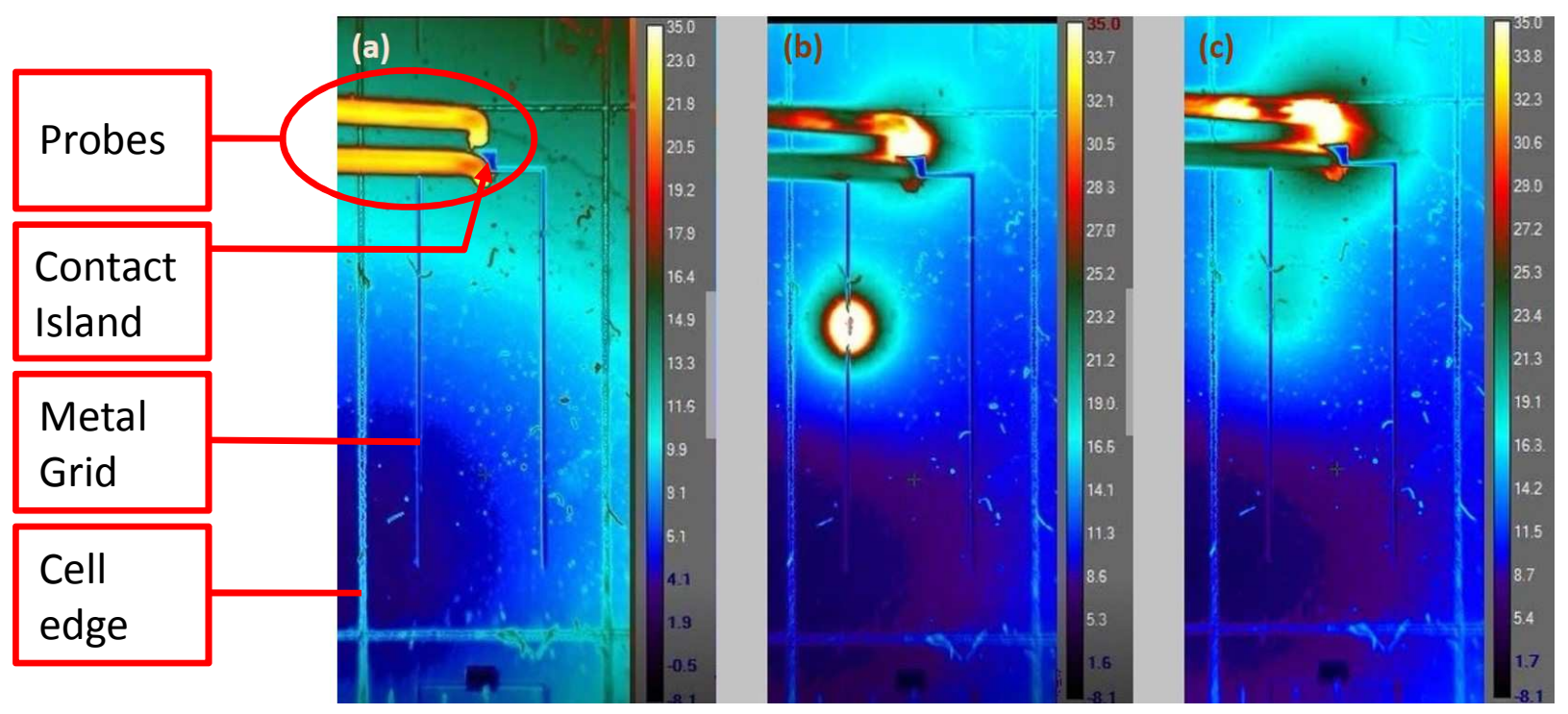

Fig. 7. IR camera images of a solar cell during dark IV characterization: (a) the IR image at low currents (b) created hot spot after reaching breakdown voltage (c) change in position of hot spot from underneath metal finger to contact probe while high current is passing through the solar cell - the size of the cell is $10 \mathrm{~mm} \times 5 \mathrm{~mm}$

image during dark IV measurements on some comparable cells from a different batch.

Three different stages of a real time movie made with an IR camera for an individual cell is shown in fig. 7. Note that the temperatures are not to scale and only function as an indication of temperature differences. The corresponding IV graph of the cell that acted in the movie is shown in fig. 8 . Fig. 7a, shows the cell at forward bias and low currents as indicated with $[\mathrm{A}]$ in the IV graph of the corresponding measurement (fig. 8). The contact probes, metal grid and cell edge are indicated in the picture of fig. 7a. The spacing of the probes is slightly bigger than the contact island. It can therefore be seen that one of the two probes is located just next to the contact island.

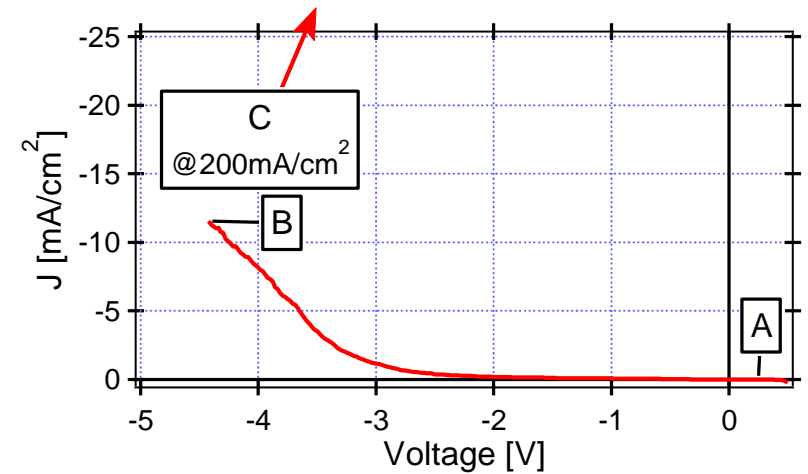

Fig. 8. JV graph of cell used for live IR imaging. Points indicated in the graph: A low current forward bias operation, B breakdown voltage point and $\mathrm{C}$ compliance current of $200 \mathrm{~mA} / \mathrm{cm} 2$ after breakdown was reached.

With increasing reverse bias voltage, the breakdown voltage was reached. The moment the breakdown appeared is shown by the creation of a hotspot underneath the metal grid in fig. $7 \mathrm{~b}$. This moment is indicated in the IV graph of fig. 8 with
[B]. Both real time IR images and IV measurements displayed that the creation of this hotspot was instantaneous. The moment the hotspot appeared, the current jumped to the current limit (compliance current) set on Keithley (200 $\mathrm{mA} / \mathrm{cm}^{2}$ ) indicated with $[\mathrm{C}]$ in fig. 8. After this incident, the set compliance current of $200 \mathrm{~mA} / \mathrm{cm}^{2}$ was still being injected. During the remaining time of the programmed IV sweep high current $\left(200 \mathrm{~mA} / \mathrm{cm}^{2}\right)$ was injected while in the thermal image, the initial hotspot underneath the metal grid disappeared and a new hotspot emerged. This new spot was formed in the area where the probe was injecting the compliance current into the TCO as seen in fig. 7c.

The movie with IR camera revealed two distinctly different mechanisms for shunt formation. First, the formation of a large shunt underneath the metal grid due to the instantaneous formation of a very intense but short living event. Second, the big contact probe injecting high current in the TCO, led to a large rise in temperature, causing the formation of a second shunt. Heat at the second hotspot can be generated both as a result of high current density in TCO and due to contact resistance between probe and TCO. One possible explanation could be that the shunt created underneath the metal grid has such a low resistance that it is not dissipating as much heat, compared to the higher resistive current path at the contact island. However it seems that both shunts are unique for cells equipped with a metal grid even if the heat generated from the contact probes could likely have been prevented with narrower probe spacing or bigger contact islands.

\section{CONCLUSIONS}

The susceptibility of non-encapsulated gridded CIGS solar cells to reverse bias voltages was monitored with an innovative experimental setup. Two test protocols have been 
defined to study the effect of exposure to reverse bias voltages and the recuperation due to consecutive light soaking. During the first test protocol, the effect of elevating reverse bias on the performance of gridded solar cells has been studied by stepwise increase of the absolute value of negative voltages in the dark, until $-1.5 \mathrm{~V}$ no significant change has happened, while a slow decrease of electrical parameters was observed after exposure to $-2 \mathrm{~V}$. This decline of electrical parameters continued for increasing negative voltages after exposure to $-3 \mathrm{~V}$ when five out of six cells broke down permanently. For the second test protocol, the effect of exposure to a constant negative voltage on the performance of gridded CIGS solar cells has been studied. It was demonstrated that the duration that cells are exposed to negative bias has a considerable impact on loss of performance. This is due to the fact that none of the cells exposed for 60 seconds to $-1.5 \mathrm{~V}$ degraded during the elevating reverse bias test. On the other hand, cells longer exposed to $-1.5 \mathrm{~V}$ bias during the constant negative voltage suffered from severe degradation. Results from light soaking after exposure to reverse bias showed a recuperation of performance for all non-fatally shunted cells. This recuperation could be an indication that besides the formation of ohmic shunts another mechanism might play a role when non-encapsulated gridded CIGS cells are exposed to a reverse bias.

Real time videos made with an IR camera revealed that shunts are created rapidly. However, for the metallisation design used in combination with the contact probes, additional damage was made by the heat generated by the injection of current by the contact probe. It is noteworthy that the hotspots found with LIT mainly appeared underneath the metal grid and did not form the typical wormlike defects that are sometimes observed in thin film modules after exposure to (partial) shading.

It should be noted that large differences were observed between the solar cells, which were deposited on one substrate. Therefore, more experiments and statistics are needed for further conclusions.

In the future, the influence of variations of other parameters (i.e. applied current instead of voltage, as well as applying different light intensities) can be tested via the experimental setup described in this study. More insight in the failure mechanisms can be obtained by studying the current over time $(\mathrm{I}(\mathrm{t}))$ and IV data obtained during this and future experiments. Future studies with the setup described in this paper will help to better understand the failure mechanism and hopefully mitigate damages due to partial shading in CIGS cells and monolithically interconnected modules.

\section{REFERENCES}

[1] S. Dongaonkar, C. Deline and M. A. Alam, "Performance and Reliability Implications of Two Dimensional Shading in Monolithic Thin Film Photovoltaic Modules," IEEE Journal Of Photovoltaics, vol. 3 no. 4, pp. 1367-1375, 2013.
[2] S. Silvestre, A. Boronat, A. Chouder, "Study of bypass diodes configuration on PV modules," Applied Energy, vol. 86, pp. 1632-1640, 2009.

[3] T. J. Silverman, L. Mansfield, I. Repins and S. Kurtz, "Damage in Monolithic Thin-Film Photovoltaic," IEEE Journal Of Photovoltaics, vol. 6 no. 5, pp. 1333-1338, 2016.

[4] T. J. Silverman, M. G. Deceglie, C. Deline and S. Kurtz, "Partial Shade Stress Test for Thin-Film Photovoltaic Modules," in Proc. SPIE 9563, Reliability of Photovoltaic Cells, Modules, Components, and Systems VIII, 95630F (September 23, 2015), 2015.

[5] N. G. Dhere, E. Schneller and A. Kaul, "Effect of shading on CIGS thin film photovoltaic modules," in 2015 IEEE 42nd Photovoltaic Specialist Conference, PVSC 7355707, 2015.

[6] T. J. Silverman, M. G. Deceglie, X, Sun, R. L. Garris, M. A. Alam, C. Deline and S. Kurtz, "Thermal and Electrical Effects of Partial Shade in Monolithic Thin-Film Photovoltaic Modules," IEEE Journal Of Photovoltaics, vol. 5 no. 6, pp. 1742-1747, 2015.

[7] P. O. Westin, U. Zimmermann, L. Stolt and M. Edoff, "Reverse Bias Damage in CIGS modules," in 24th European Photovoltaic Solar Energy Conference, pp. 2967-2970, 2009.

[8] P. Mack, T. Walter, R. Kniese, D. Hariskos, and R. Schäffler, "Reverse Bias and Reverse Currents in CIGS Thin Film Solar Cells and Modules," 23rd European Photovoltaic Solar Energy Conference and Exhibition, pp. 2156-2159, 2008.

[9] P. Szaniawski, J. Lindahl, T. Törndahl, U. Zimmermann and M. Edoff, "Light-enhanced reverse breakdown in $\mathrm{Cu}(\mathrm{In}, \mathrm{Ga}) \mathrm{Se}_{2}$ solar cells," Thin Solid Films, vol. 535, pp. 326-330, 2013.

[10] S. Dongaonkar, M. A. Alam, Y. Karthik, S. Mahapatra, D. Wang and M. Fei, "Identification, characterization, and implications of shadow degradation in thin film solar cells," in 2011 IEEE 49th International Reliability Physics Symposium, IRPS 5784535, pp. 5E.4.1-5E.4.5, 2011. 